ORIGINAL PAPER

\author{
Wolf E. Arntz $\cdot$ Sven Thatje $\cdot$ Katrin Linse \\ Conxita Avila $\cdot$ Manuel Ballesteros \\ David K. A. Barnes · Thérèse Cope \\ Francisco J. Cristobo · Claude De Broyer \\ Julian Gutt · Enrique Isla · Pablo López-González \\ Américo Montiel · Tomás Munilla \\ Alfonso A. Ramos Esplá · Michael Raupach \\ Martin Rauschert · Estefanía Rodríguez · Núria Teixidó

\section{Missing link in the Southern Ocean: sampling the marine benthic fauna of remote Bouvet Island}

Received: 18 January 2005/ Revised: 30 June 2005/ Accepted: 1 July 2005/Published online: 22 September 2005

(C) Springer-Verlag 2005

AbstractBouvet (Bouvetøya) is a geologically young and very remote island just south of the Polar Front. Here we report samples taken during the RV "Polarstern" cruise ANTXXI/2 on 3 days in November 2003 and January 2004. This work was part of SCAR's EASIZ programme and intended, by providing data on the marine fauna of this "white gap" in the Atlantic sector of the Southern Ocean, to contribute to identifying the role of Bouvet in the faunal exchange between the Suband high Antarctic. While this goal demands extensive

W. E. Arntz · J. Gutt · A. Montiel

Alfred Wegener Institute for Polar and Marine Research, P.O. Box 120-161, 27515 Bremerhaven, Germany

S. Thatje $(\square)$

National Oceanography Centre, School of

Ocean and Earth Science,

University of Southampton, European Way,

Southampton, SO14 3ZH, UK

E-mail: svth@noc.soton.ac.uk

K. Linse $\cdot$ D. K. A. Barnes · T. Cope

British Antarctic Survey,

Natural Environmental Research Council, High Cross,

Madingley Road, Cambridge, CB3 0ET, UK

C. Avila

Centre d'Estudis Avançats de Blanes CEAB-CSIC,

c/ Accés a la cala Sant Francesc 14,

17300 Blanes Girona, Catalunya, Spain

M. Ballesteros

Dept. Biología Animal, Facultad de Biología,

Universidad de Barcelona, Av. Diagonal 645,

08028 Barcelona, Spain

\section{F. J. Cristobo}

Estación de Bioloxía Mariña da Graña,

Universidade de Santiago de Compostela,

Rúa da Ribeira 1, 15590 A Graña

Ferrol, Galicia, Spain molecular analysis of the material sampled (future work), a checklist of the samples and data at hand widens the faunal and environmental inventory substantially. We suggest some preliminary conclusions on the relationship of Bouvet Island's fauna with that of other regions, such as Magellanic South America, the Antarctic Peninsula, and the high Antarctic Weddell Sea, which have been sampled previously. There seem to be different connections for individual higher taxa rather than a generally valid consistent picture.

\section{De Broyer}

Dept. des Invertébrés (Carcinologie),

Institut royal des Sciences Naturelles de Belgique,

Rue Vautier 29, 1000 Brussels, Belgium

E. Isla $\cdot$ E. Rodríguez $\cdot$ N. Teixidó

Institut de Ciències del Mar, Passeig Marítim de la Barceloneta, 37-49, 08003 Barcelona, Spain

P. López-González · E. Rodríguez

Dpto. Fisiología y Zoología, Facultad de Biología,

Universidad de Sevilla,

Reina Mercedes 6, 41012 Sevilla, Spain

T. Munilla

Departamento de Biología Animal,

Universidat Autónoma de Barcelona, 08193

Bellaterra, Barcelona, Spain

A. A. Ramos Esplá

Dpto. Ciencias del Mar y Biología Aplicada,

Universidad de Alicante, Campus de Sant Vicent del Raspeig, 03080 Alicante, Spain

M. Raupach

Lehrstuhl für Spezielle Zoologie, ND 05/577,

Ruhr-Universität Bochum,

Universitätsstr. 150, 44780 Bochum, Germany

M. Rauschert

Bruno-H.-Bürgel-Allee 31, 15732 Eichwalde, Germany 


\section{Introduction}

Bouvet Island is one of the most isolated places on Earth. At $54^{\circ} 26^{\prime} \mathrm{S}, 3^{\circ} 24^{\prime} \mathrm{E}$ on the Mid-Atlantic Ridge between Africa and Antarctica, south of the Polar Front, it is further from land than any other island. As a result of its position in the principal track of the lows and gales continuously passing around the Antarctic continent and at the northern margin of the Antarctic pack ice, its marine ecosystem has received little attention since its discovery in 1739 . Benthic marine fauna in the vicinity of the island was collected on three major expeditions: the "Deutsche Tiefsee Expedition" 1898-1899 (e.g. Thiele 1904, 1925; Thiele and Jaeckel 1931), the "Discovery" Expeditions 1926-1937 (Powell 1951; Dell 1964), and the Norwegian Antarctic Expeditions 1927-1930 (Bergenhayn 1937; Soot-Ryen 1951), whereas in more recent times the emphasis has been on plankton research (Fevolden 1980; Perissinotto et al. 1992). The $50 \mathrm{~km}^{2}$ ice-covered, volcanically active island under Norwegian authority has mainly attracted study because of the unusual geologic and volcanic properties (Furnes and Løvlie 1978), reflecting its young geological age of just $1 \mathrm{Ma}$ (Prestvik and Winsnes 1981). What sparse biological information exists refers to the island's rich coastal fauna of seals and seabirds (Haftorn et al. 1981), its terrestrial vegetation (Holdgate et al. 1968; Engelskjön 1981), and the physiological cold adaptation of micro-arthropods (Somme 1986).

One of the major tasks of the EASIZ (Ecology of the Antarctic Sea Ice Zone) programme of SCAR has been to compare areas of the Southern Ocean influenced by pack ice with those without pack ice cover. Within this programme, considerable data have been assembled from work in and across the Magellan region, the Scotia Arc, the Antarctic Peninsula, and the high Antarctic Weddell Sea (e.g. Arntz and Gorny 1996; Arntz and Gutt 1997, 1999; Arntz and Ríos 1999; Gutt et al. 2000; Arntz and Brey 2001, 2003; Arntz et al. 2005). However, up to the cruise reported here, Bouvet Island and its surrounding area remained faunistically almost unknown. Bouvet is particularly important because of its position in the Antarctic Circumpolar Current (ACC) and the Weddell Gyre (Linse 2005; Thatje et al. 2005b), suggesting a potentially strategic role in the faunal exchange among the various regions and across the Polar Front. Pelagic larvae and drift stages must predominantly be transported eastward by the ACC or by vehicles travelling in it, such as pumice, drifting algae, driftwood, and plastic debris, often over enormous distances (Barber et al. 1959; Coombs and Landis 1966; Helmuth et al. 1994; Beu et al. 1997; O Foighil et al. 1999; Barnes 2002). However, deep-sea migration of eurybathic species (Brey et al. 1996; Thatje et al. 2005a), transport via mesoscale eddies (Thatje and Fuentes 2003; Glorioso et al. 2005; Gayoso and Podestá 1996), or hitch-hiking on migrant megafauna (Barnes et al. 2004) seems to represent the only means of return. There may, however, exist a Weddell Gyre-East Wind Drift connection, in which Bouvet might play a major role. To speculate on the existence of such potential zoogeographic links requires phylogenetic analyses and careful interpretation of the samples by taxon. Our attempt is a synoptic overview on the local marine fauna encountered, which is also a contribution to the understanding and cataloguing of Southern Ocean biodiversity (Clarke and Johnston 2003).

\section{Work at sea and methodology}

Sampling near Bouvet Island (54'26'S, $\left.3^{\circ} 24^{\prime} \mathrm{E}\right)$ was carried out on the 24th and 25th of November 2003, and at nearby Spiess Seamount (54 $44^{\prime}$ S, $\left.0^{\circ} 07^{\prime} \mathrm{E}\right)$ on January 11, 2004 (Arntz and Brey 2005). We used a $1.5 \mathrm{~m} \mathrm{A-}$ gassiz trawl (AGT) with the $(10 \mathrm{~mm})$ small, narrowmeshed (1 mm) Rauschert dredge (RD) tied behind it, baited traps, and an UW camera (photosled) (for station data, see Arntz and Brey 2005). Randomly, 51 subsamples were taken from the AGT catches for quantitative analysis. At the Spiess Seamount we used a normal (3 m) Agassiz trawl again with an RD tied behind, a stone dredge, and a photosled (for station data, see Arntz and Brey 2005). In addition to the AGT subsamples, taxonomists randomly collected invertebrate species from the remaining AGT sample on deck, which contributed to the overall assessment of benthic diversity given in this article (Table 2). CTD casts were performed with a SeaBird 911plus series from the surface to an approximate distance of $1-5 \mathrm{~m}$ above the seabed (Fig. 1).

Faunistic resemblance between stations (pooled AGT and RD data) was measured by Bray-Curtis similarities (Bray and Curtis 1957) of taxon presence and absence data using PRIMER 5 (Clarke and Warwick 2001). Non-metric multidimensional scaling was applied to resemblance the data to reflect faunistic similarities in a two-dimensional scale.

\section{Preliminary results}

\section{Hydrography}

Bouvet was mainly surrounded by open water at our sample dates in November, with a number of icebergs and growlers stranded close to the island and occasional large icebergs drifting by. The temperature of surface waters around the island was about -0.6 to $-0.7^{\circ} \mathrm{C}$ (Fig. 2left), and surface salinity was between 34.1 and 34.2 PSU, with a mixed layer extending to $160-200 \mathrm{~m}$ on 24th November but only to about $70 \mathrm{~m}$ at the northernmost station on 25th November (Fig. 2right). Below the pycnocline, warmer and saltier water extended at least to a water depth of $600 \mathrm{~m}$. 
Fig. 1 Location of AGT, photosled (FTS), and CTD sampling sites at Bouvet Island. Grey circle AGT; black square FTS; open triangle CTD; grey lines depth contours: $100 \mathrm{~m}$, $200 \mathrm{~m}, 300 \mathrm{~m}, 400 \mathrm{~m}, 500 \mathrm{~m}$, $1,000 \mathrm{~m}$

Fig. 2 CTD graphs from Bouvet Island. Deep-water station PS65/021-1 (left). Shallow-water station PS65 018-1 (right)
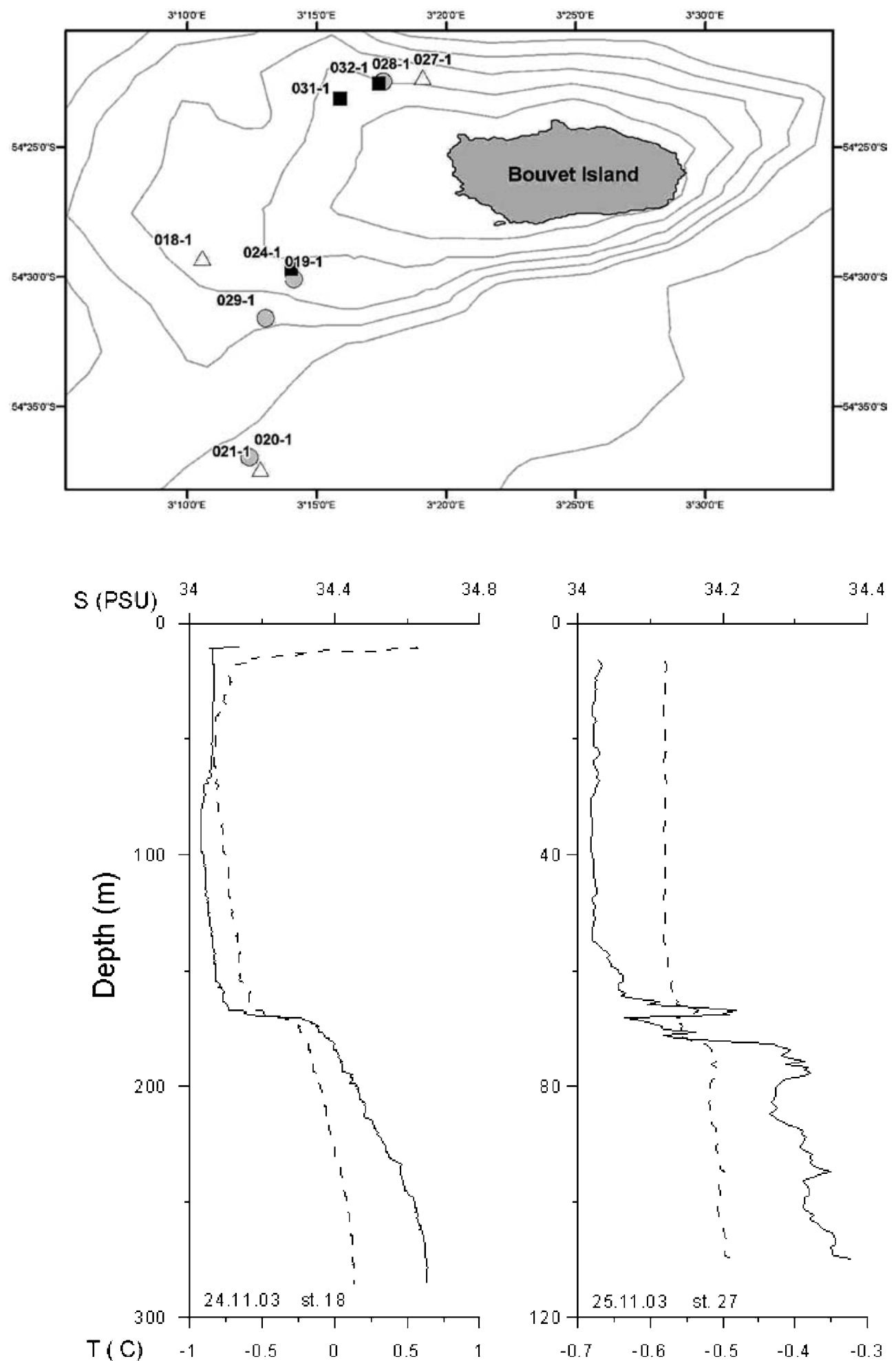

Bathymetry and related gear success

The seafloor around Bouvet (four AGT stations, 100$550 \mathrm{~m}$ ) was not particularly rugged (Fig. 3). Typically, the volcanic bottom topography was smooth, overlain by a thin layer of coarse sand or lava pebbles (in AGT 3 sample). All four Agassiz trawls were retrieved without damage and successfully caught a variety of benthos (Fig. 4a-d). Conversely, the bottom topography at the Spiess Seamount was extremely varied, with steep peaks and crevices. Despite the use of hydrosweep, the net of our second Agassiz trawl was damaged and the RD removed. Our first Agassiz trawl had been retrieved full of stones. For this reason, a stone dredge was then 
deployed. The photosled yielded full picture transects at both sites (for example, see Fig. 3).

Benthic fauna: description and diversity

\section{General description of sites at Bouvet Island and Spiess Seamount}

In order to get a preliminary impression of the faunistic diversity at different depth ranges, photographic images were taken either from the AGT catch on board (Fig. 4) or in situ by the photosled (Fig. 3; see also Gutt et al. 2005). The taxonomic composition and ecological importance of taxa in catches at Bouvet based on AGT samples are summarised in Table 1. In general, echinoderms, in particular ophiuroids, were highly abundant except echinoids, which were almost absent. Compared with the E Weddell Sea (high Antarctic), the structural three-dimensionality of the benthic communities was low partly because of the lack of large sponges that act as raised substrates for other taxa such as crinoids and
Fig. 3 Photosled survey at Bouvet Island: station PS65 022-1, $550 \mathrm{~m}$ (pic 051); station PS65/031-1, 120 m (pic 034); station PS65/024-1, $250 \mathrm{~m}$ (pic 107); station PS65/024-1, $250 \mathrm{~m}$ (pic 107b); station PS65/343-1, $460 \mathrm{~m}$ (pic 50); at Spiess

Seamount: station PS65/343-1, $460 \mathrm{~m}$ (pic 68)
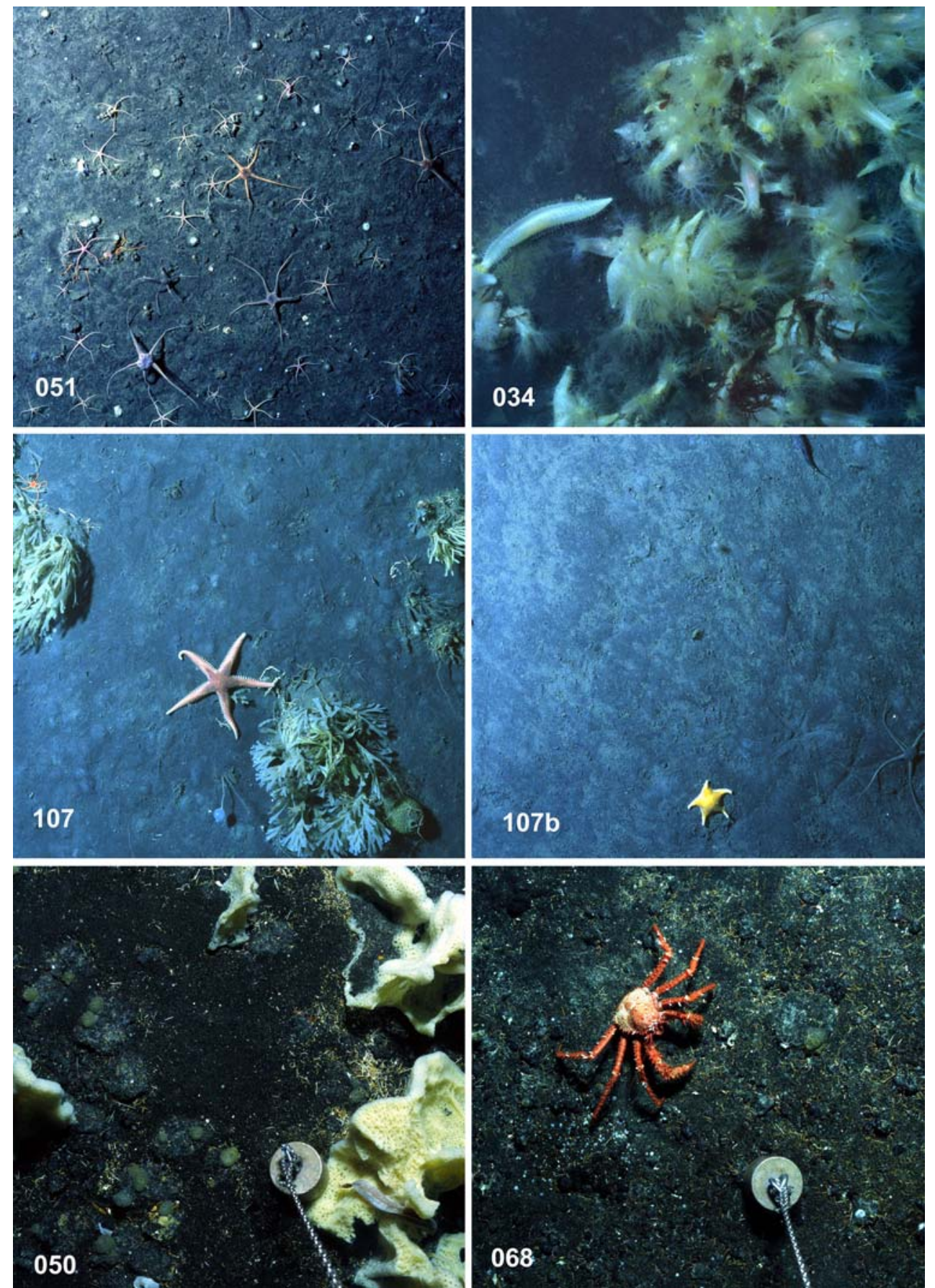

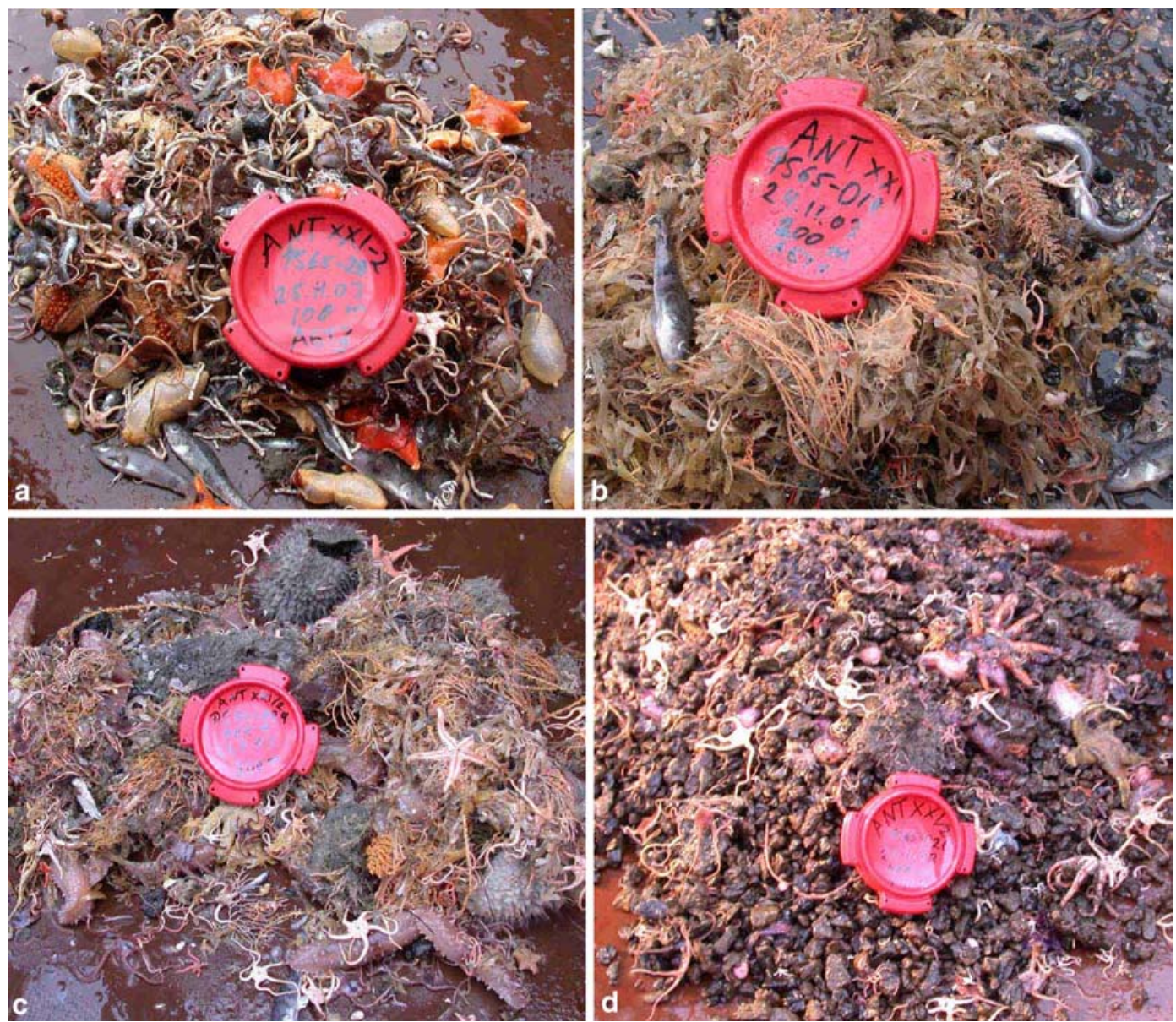

Fig. 4 Visual quality and quantity assessment of Agassiz trawl catches at Bouvet Island a $130 \mathrm{~m}$ Station PS65/028-1, b $250 \mathrm{~m}$ Station PS65/019-1, c 370 m Station PS65/029-1, d 550 m Station PS65/020-1

holothurians. Red macroalgae (Rhodophyta) and hydroids were most abundant at $130 \mathrm{~m}$, erect, flexible cheilostome bryozoans (mainly Austroflustra vulgaris) at $250 \mathrm{~m}$, and some large sponges and gorgonians added complexity to the assemblage at $370 \mathrm{~m}$. Other ecologically important elements at $130 \mathrm{~m}$ were serpulid polychaetes, small amphipods (such as caprellids), small pycnogonids, and small notothenioid fish (mostly Lepidonotothen larseni, one L. kempi).

Macrozoobenthic composition based on relative abundances revealed a strong increase of echinoderm dominance (mainly ophiuroids, see Tables 1,2) with increasing water depth. Polychaetes and arthropods (especially amphipods) dominated the shallower water at $130 \mathrm{~m}$ depth (Fig. 5), but became less abundant in deeper waters.

The catches of the small RD resembled the AGT ones in larger macrofauna, e.g. echinoderms, but added taxon presence for small macrofauna such as peracarid crustaceans (Tables 1, 2).

No zoarcid fish or lithodid decapods were caught in waters around Bouvet Island; however, they might as well occur in deeper water. No other reptant decapods

were detected and only three caridean (hippolytid) shrimp species and the deep-water shrimp Nematocarcinus lanceopes, typical of the Southern Ocean, were found. Several typical high Antarctic faunal elements were missing, including the large epimeriid and eusirid amphipods, large serolid and Glyptonotus isopods, large pycnogonids, muricid and kelliid molluscs, and, with one exception, large polynoid and aphroditid polychaetes. Isopods were rare and most of them (except Antarcturus) were very small.

\section{Systematic diversity assessment}

The collected benthic taxa were separated by class and sent to specialists for further taxonomic identifications. The following represents the species and diversity assessments given the latest stage of identifications (Table 2).

Porifera The collected sponges were dominated by hexactinellids and demosponges, and one calcareous species of the order Clathrinida was collected. At the moment, Calcarea are accounted as rare in Antarctica with only a few described species. The species Tedania (Tedaniopsis) tantula and Tentorium sp. were found at two sites whereas the other four species (Mycale (Oxymycale) acerata, Polymastia invaginata, Pseudosuberites sp., and 
Table 1 Taxonomic composition and ecological importance of taxa in AGT catches at Bouvet Island

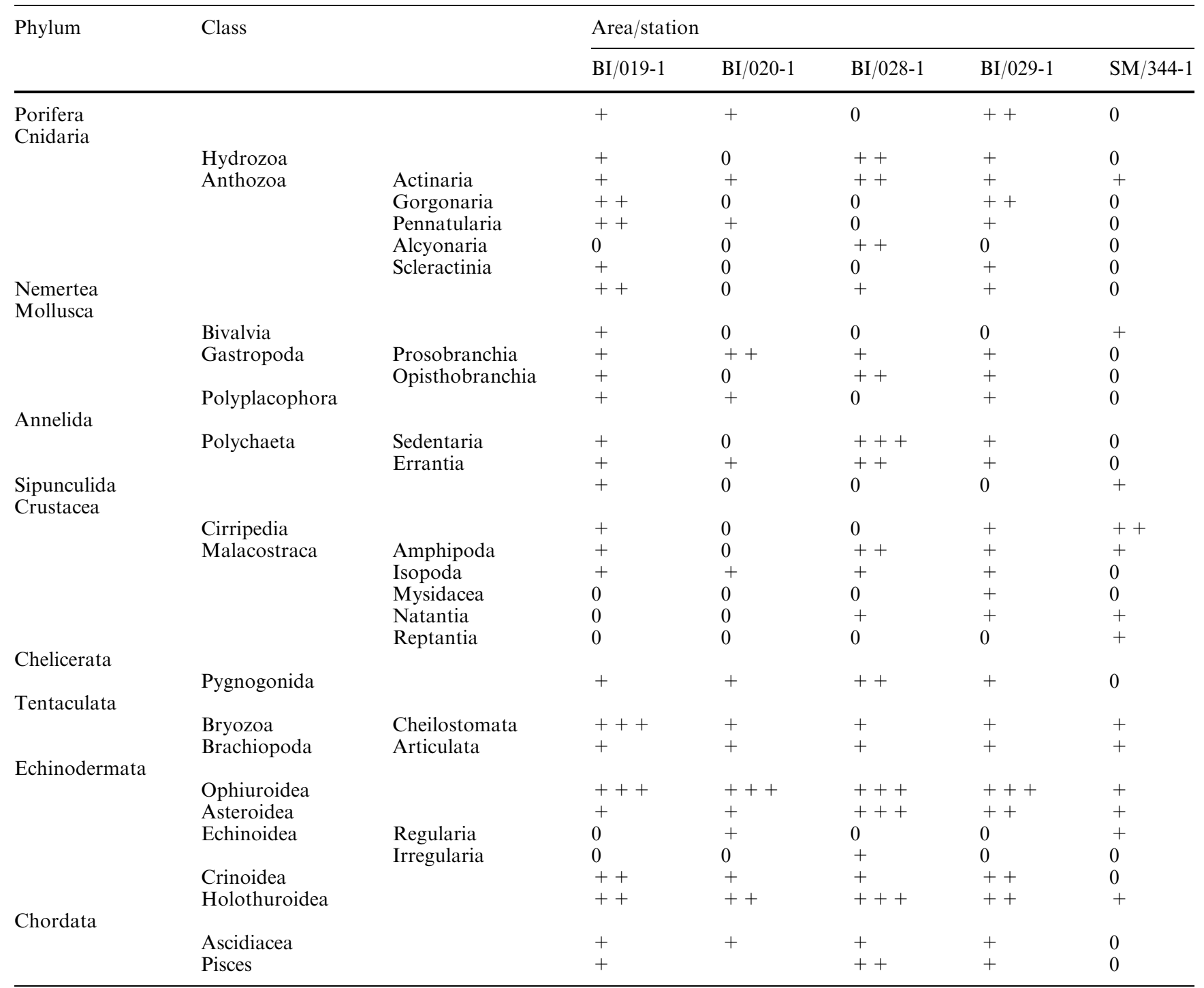

BI Bouvet Island, SM Spiess Seamount, 0 absent, + rare, ++ common, +++ very abundant/dominant

Bubaris sp.) were found at one site only. T. (Tedaniopsis) tantula (Kirkpatrick 1907) and $M$. (Oxymycale) acerata (Kirkpatrick 1907) are circumAntarctic species. M.O. acerata has also been found off the Argentinean and Brazilian coasts between $56^{\circ}-40^{\circ} \mathrm{S}$ and $39^{\circ} \mathrm{S}$, and the Chilean coast between $33^{\circ}$ and $35^{\circ} \mathrm{S}$, in the Juan Fernández Islands (Desqueyroux and Moyano 1987).

Cnidaria The dominant cnidarian class was anthozoans and within them actininans while hydrozoans were present but rare. Prior to the current sampling reported in this series of articles on Bouvet Island's marine fauna, just five species of actinians were known from Bouvet waters. Ten species are added now, which still need to be identified to species level, increasing the number of known actinians for the region to 13 species. The actinian species/morphospecies found at Bouvet Island and Spiess seamount show biogeographic affinities with both Subantarctic South America and the high Antarctic Weddell Sea fauna. About $40 \%$ of actinian species found show a circum-Antarctic distribution. Scleractinia and octocorals, such as Ascolepis sp., Thouarella sp,. and Primnoella sp., were present and the latter common at stations between 250 and $380 \mathrm{~m}$.

Nemerteans Nemerteans were rare in the samples and only one, red coloured, yet unidentified species was found.

Annelida-Polychaeta The polychaete material obtained from the AGT subsamples revealed 14 species, which have been identified so far at the genus level only. The genera found indicate that the Bouvet polychaete fauna may show biogeographic affinities with both Subantarctic South America and the Weddell Sea fauna. 
Table 2 Taxon-station list for the benthos collected at Bouvet Island and Spiess Seamount

\begin{tabular}{|c|c|c|c|c|c|c|c|}
\hline \multirow[t]{2}{*}{ Taxon } & \multirow[t]{2}{*}{ Species } & \multicolumn{6}{|c|}{ Area/station/depth } \\
\hline & & $\begin{array}{l}\mathrm{BI} / \\
019-1 / \\
250 \mathrm{~m}\end{array}$ & $\begin{array}{l}\mathrm{BI} / \\
020-1 / \\
550 \mathrm{~m}\end{array}$ & $\begin{array}{l}\mathrm{BI} / \\
028-1 / \\
130 \mathrm{~m}\end{array}$ & $\begin{array}{l}\mathrm{BI} / \\
029-1 / \\
380 \mathrm{~m}\end{array}$ & $\begin{array}{l}\mathrm{SM} / \\
344-1 / \\
580 \mathrm{~m}\end{array}$ & $\begin{array}{l}\mathrm{SM} / \\
347-1 / \\
400 \mathrm{~m}\end{array}$ \\
\hline \multicolumn{8}{|l|}{ Porifera } \\
\hline & $\begin{array}{l}\text { Clathrinida sp. } \\
\text { Bubaris sp. } \\
\text { Mycale }(O .) \text { acerata } \\
\text { Polymastia invaginata } \\
\text { Pseudosuberites sp. } \\
\text { Tedania }(T .) \text { tantula } \\
\text { Tentorium } \mathrm{sp} . \\
\text { Undetermined }\end{array}$ & + & $\begin{array}{l}+ \\
+ \\
+ \\
+\end{array}$ & + & $\begin{array}{l}+ \\
+\end{array}$ & + & \\
\hline \multicolumn{8}{|l|}{ Cnidaria } \\
\hline Hydrozoa & Undetermined & & & + & & & \\
\hline Anthozoa & $\begin{array}{l}\text { Alcyonaria sp. } \\
\text { Ascolepis sp. } \\
\text { Thouarella sp. } \\
\text { Primnoidae sp. } \\
\text { Actinostola sp. } \\
\text { Actinostolidae sp. } 1 \\
\text { Actinostolidae sp. } 2 \\
\text { Actinostolidae sp. } 3 \\
\text { Capnea georgiana } \\
\text { Hormathiidae sp. } \\
\text { Glyphoperidium bursa } \\
\text { Pycnanthus sp. } \\
\text { Stomphia selaginella } \\
\text { Isotealia antarctica } \\
\text { Flabellum } \text { sp. }\end{array}$ & $\begin{array}{l}+ \\
+ \\
+ \\
+ \\
+ \\
+ \\
+ \\
+\end{array}$ & + & $\begin{array}{l}+ \\
+ \\
+\end{array}$ & $\begin{array}{l}+ \\
+\end{array}$ & + & \\
\hline \multicolumn{8}{|l|}{ Annelida } \\
\hline 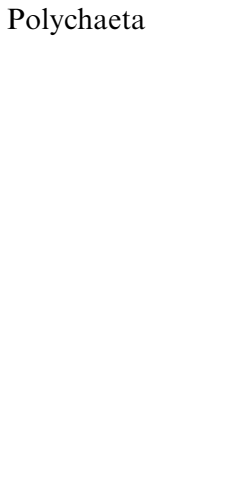 & $\begin{array}{l}\text { Phyllocomus sp. } \\
\text { Laetmonice sp. } \\
\text { Flabelligera } \mathrm{sp} . \\
\text { Aglaophamus } \mathrm{sp} . \\
\text { Barrukia } \mathrm{sp} . \\
\text { Eulagisca } \mathrm{sp} . \\
\text { Eunoe } \mathrm{sp} . \\
\text { Harmothoe spp. } \\
\text { Hermadion sp. } \\
\text { Polyeunoa } \mathrm{sp} . \\
\text { Amphiglena } \mathrm{sp} . \\
\text { Serpula } \mathrm{sp} . \\
\text { Autolytus } \mathrm{sp} . \\
\text { Trypanosyllis } \mathrm{sp} . \\
\text { Pista } \mathrm{sp} .\end{array}$ & $\begin{array}{l}+ \\
+ \\
+ \\
+ \\
+ \\
+\end{array}$ & + & $\begin{array}{l}+ \\
+ \\
+ \\
+ \\
+\end{array}$ & $\begin{array}{l}+ \\
+ \\
+ \\
+\end{array}$ & + & \\
\hline \multicolumn{8}{|c|}{$\begin{array}{l}\text { Mollusca (see Linse 2005) } \\
\text { Gastropoda }\end{array}$} \\
\hline Prosobranchia & Puncturella conica & & & & & + & + \\
\hline Opisthobranchia & $\begin{array}{l}\text { Austrodoris kerguelensis } \\
\text { Bathyberthella antarctica } \\
\text { Cuthona sp. } \\
\text { Doto sp. } \\
\text { Notaeolidia depressa } \\
\text { Philine alata } \\
\text { Tritonia challengeriana } \\
\text { Tritonia sp. }\end{array}$ & $\begin{array}{l}+ \\
+ \\
+ \\
+\end{array}$ & & $\begin{array}{l}+ \\
+ \\
+ \\
+\end{array}$ & $\begin{array}{l}+ \\
+ \\
+ \\
+ \\
+\end{array}$ & & \\
\hline Chelicerata & & & & & & & \\
\hline Pygnogonida & $\begin{array}{l}\text { Ammothea carolinensis } \\
\text { Colossendeis robusta } \\
\text { Nymphon australe } \\
\text { Pallenopsis hodgsoni }\end{array}$ & $\begin{array}{l}+ \\
+\end{array}$ & & + & $\begin{array}{l}+ \\
+\end{array}$ & & \\
\hline Nemerteans & Antarctonemertes sp. & + & + & + & + & & \\
\hline
\end{tabular}


Table 2 (Contd.)

\begin{tabular}{|c|c|c|c|c|c|c|c|}
\hline \multirow[t]{2}{*}{ Taxon } & \multirow[t]{2}{*}{ Species } & \multicolumn{6}{|c|}{ Area/station/depth } \\
\hline & & $\begin{array}{l}\mathrm{BI} / \\
019-1 / \\
250 \mathrm{~m}\end{array}$ & $\begin{array}{l}\mathrm{BI} / \\
020-1 / \\
550 \mathrm{~m}\end{array}$ & $\begin{array}{l}\mathrm{BI} / \\
028-1 / \\
130 \mathrm{~m}\end{array}$ & $\begin{array}{l}\mathrm{BI} / \\
029-1 / \\
380 \mathrm{~m}\end{array}$ & $\begin{array}{l}\mathrm{SM} / \\
344-1 / \\
580 \mathrm{~m}\end{array}$ & $\begin{array}{l}\mathrm{SM} / \\
347-1 / \\
400 \mathrm{~m}\end{array}$ \\
\hline \multicolumn{8}{|l|}{ Sipunculida } \\
\hline & Golfingia sp. & + & & & & & + \\
\hline \multirow{60}{*}{$\begin{array}{l}\text { Crustacea } \\
\text { Cirripedia } \\
\text { Tanaidacea } \\
\text { Amphipoda }\end{array}$} & Arcoscalnellum houvieri & & & & & & \\
\hline & $\begin{array}{l}\text { Arcoscalpellum bouvlert } \\
\text { Apseudes spectabilis }\end{array}$ & $\begin{array}{l}+ \\
+\end{array}$ & & & + & & + \\
\hline & Giganopsis squamosa & & & + & & & \\
\hline & Atyloellatribinicuspidata sp. n. & + & & + & + & & \\
\hline & Atylopsis fragilis & + & + & + & + & & \\
\hline & Atylopsis megalops & & + & + & & & \\
\hline & Atylopsis orthodactylus & & & + & + & & \\
\hline & Harpinioides drepanocheir & + & & & & & \\
\hline & Oradarea rossi & & & + & & & \\
\hline & Oradarea walkeri & & & + & & & \\
\hline & Prostebbingia brevicornis & & & & + & & \\
\hline & Prostebbingia sp. & & & + & & & \\
\hline & Schraderia gracilis & + & & + & + & & \\
\hline & Echiniphimedia hodgsoni & & & + & & & \\
\hline & Gnathiphimedia sp. n. 1 & + & & & & & \\
\hline & Gnathiphimedia cf. mandibularis & & & + & & & \\
\hline & Gnathiphimedia mandibularis & + & & + & + & & \\
\hline & Gnathiphimedia sp. & + & & & & & \\
\hline & Gnathiphimedia sp. n. 2 & & & + & + & & \\
\hline & Leucothoe spinicarpa & & & + & + & & \\
\hline & Liljeborgia georgiana & + & & + & + & & \\
\hline & Liljeborgia sp. n. 1 & & & + & & & \\
\hline & Liljeborgia sp. & + & + & + & & & \\
\hline & Liljeborgia sp. n. 2 & & & & + & & \\
\hline & Arisatsis sp. & & & + & & & \\
\hline & Lysianassoidea sp. n. 1 & + & & + & + & & \\
\hline & Hippomedon kergueleni & & & & + & & \\
\hline & Orchomenopsis cavimanus & & & + & & & \\
\hline & Pachychelium nichollsi & & & + & & & \\
\hline & Lysianassoidea sp. n. 2 & & + & + & & & \\
\hline & Tryphosella $\mathrm{sp}$. & + & + & + & + & & \\
\hline & Melphidippa antarctica & + & & + & & & \\
\hline & Oedicerotidae sp. & & & + & & & \\
\hline & Pagentina genarum & & & + & & & \\
\hline & Pardaliscidae sp. & & & & + & & \\
\hline & Parepimeria crenulata & + & & + & & & \\
\hline & Seba antarctica & & & & + & & \\
\hline & Stegocephalidae sp. & & & + & & & \\
\hline & Antatelson walkeri & & & + & & & \\
\hline & Stenothoidae sp. & & & & + & & \\
\hline & Mesoproboloides sp. $\mathrm{n}$. & + & & & & & \\
\hline & Metopoides leptomanus & & & + & & & \\
\hline & Metopoides sp. n. 1 & & & + & + & & \\
\hline & Metopoides sp. n. 2 & & & & + & & \\
\hline & Metopoides sp. n. 3 & & & + & & & \\
\hline & Torometopa antarctica & & & + & & & \\
\hline & Torometopa sp. 1 & & & + & & & \\
\hline & Torometopa sp. 2 & & & + & & & \\
\hline & Torometopa sp. n. 1 & & & + & & & \\
\hline & Torometopa sp. n. 2 & + & & + & & & \\
\hline & Stilipedidae sp. & & & & + & & \\
\hline & Gamaropsis serricra & & & + & + & & \\
\hline & Haplocheira barbimana & & & + & + & & \\
\hline & Caprellinoides tristanensis & & & + & + & & \\
\hline & Aeginioides gaussi & + & & & & & \\
\hline & Podocerus capillimanus & & & + & & & \\
\hline & Pseudodulichia antarctica & & & & + & & \\
\hline & Ischyroceridae sp. 1 & & & & + & & \\
\hline & Ischyroceridae sp. 2 & & & + & & & \\
\hline & Jassa falcata & & & + & & & \\
\hline
\end{tabular}


Table 2 (Contd.)

\begin{tabular}{|c|c|c|c|c|c|c|c|}
\hline \multirow[t]{2}{*}{ Taxon } & \multirow[t]{2}{*}{ Species } & \multicolumn{6}{|c|}{ Area/station/depth } \\
\hline & & $\begin{array}{l}\mathrm{BI} / \\
019-1 / \\
250 \mathrm{~m}\end{array}$ & $\begin{array}{l}\mathrm{BI} / \\
020-1 / \\
550 \mathrm{~m}\end{array}$ & $\begin{array}{l}\mathrm{BI} / \\
028-1 / \\
130 \mathrm{~m}\end{array}$ & $\begin{array}{l}\mathrm{BI} / \\
029-1 / \\
380 \mathrm{~m}\end{array}$ & $\begin{array}{l}\mathrm{SM} / \\
344-1 / \\
580 \mathrm{~m}\end{array}$ & $\begin{array}{l}\mathrm{SM} / \\
347-1 / \\
400 \mathrm{~m}\end{array}$ \\
\hline & Jassa goniamera & & & + & + & & \\
\hline & Pseudericthonius gaussi & & & & + & & \\
\hline & Pseudericthonius hesperidesi & + & + & + & & & \\
\hline & Scina rattrayi rattrayi & & & + & & & \\
\hline \multirow[t]{13}{*}{ Isopoda } & Ianthopsis ruseri & + & & & & & \\
\hline & Acanthomunna spinipes & & & & + & & \\
\hline & Neojaera antarctica & & & + & & & \\
\hline & Joeropsis antarctica & + & & & & & \\
\hline & Munna sp. 1 & + & & + & & & \\
\hline & Munna sp. 2 & & & & + & & \\
\hline & Echinozone quadrispinosa & + & & + & + & & \\
\hline & Coperonus sp. & + & & + & + & & \\
\hline & Pleurogonium sp. & & & + & & & \\
\hline & Aega pushkini & & + & & + & & \\
\hline & Ceacognathia sp & + & & + & & & \\
\hline & Septemserolis septemcarinata & + & + & & + & & \\
\hline & Antarcturus oryx & + & + & + & + & & \\
\hline Mysidacea & Mysidacea sp. & & & & + & & \\
\hline \multirow[t]{4}{*}{ Natantia } & Nauticaris marionis & & & + & & & \\
\hline & Hippolytidae undet. sp. 1 & & & & + & & \\
\hline & Hippolytidae undet. sp. 2 & & & & + & & \\
\hline & Nematocarcinus lanceopes & & & & & + & \\
\hline Reptantia & Paralomis elongata sp. $\mathrm{n}$. & & & & & + & + \\
\hline \multicolumn{8}{|l|}{ Tentaculata } \\
\hline \multirow[t]{18}{*}{ Bryozoa } & Aimulosia antarctica & + & & + & & & \\
\hline & Arachnopusia inchoata & + & & + & & & \\
\hline & Austroflustra vulgaris & + & + & & + & & \\
\hline & Camptoplites bicornis & & & & + & & \\
\hline & Cellaria incula & & & & + & & \\
\hline & Cellarinella njegovanae & + & & & + & & \\
\hline & Cellarinella nutti & + & & + & & & \\
\hline & Celleporella antarctica & & & + & & & \\
\hline & Celleporella bougainvillei & & & + & & & \\
\hline & Himantozoum antarcticum & + & & & & & \\
\hline & Nematoflustra flagellata & + & + & + & + & & \\
\hline & Osthimosia bicornis & + & & & + & & \\
\hline & Osthimosia sp. & + & & + & & & \\
\hline & Polirhabdotos inclusum & + & & & & & \\
\hline & Reteporella erugata & + & & & + & & \\
\hline & Reteporella frigida & & & & + & & \\
\hline & Smittina incernicula & + & & & & & \\
\hline & Swanomia brevimandibulata & & & & + & & \\
\hline Brachiopoda & Brachiopod sp. & + & & + & & & \\
\hline Echinodermata & & & & & & & \\
\hline \multirow{9}{*}{ Asteroidea } & Odontaster sp. & + & & & & & \\
\hline & Luidia sp. & & + & & + & & \\
\hline & Porania cf. antarctica & & & + & & & \\
\hline & Acodontaster sp. & & + & & & & \\
\hline & Psilaster sp. & & & & + & & \\
\hline & Crossaster sp. & & + & & & & \\
\hline & Asteroid sp. 1 & & & + & & & \\
\hline & Asteroid sp. 2 & & & & & + & \\
\hline & Cuenotaster $\mathrm{sp}$ & & & & + & & \\
\hline Crinoidea & Undetermined & + & & + & & & \\
\hline \multirow{2}{*}{ Echinoidea } & Regularia sp. & & + & & & + & \\
\hline & Irregularia sp. & & & + & & & \\
\hline \multirow{8}{*}{ Holothuridea } & Ekmocucumis sp. & & + & & + & & \\
\hline & Ekmocucumis cf. steineni & & & + & & & \\
\hline & Dendroichiratacea & & + & & & & \\
\hline & Dendrochiratacea & + & + & & & & \\
\hline & Bathyplotes sp. & & + & & + & & \\
\hline & Bathyplotes fuscivinculum & & & & + & & \\
\hline & Psolus $\mathrm{cf}$. antarcticus & & + & & & & \\
\hline & Psolidae sp. 1 & & + & & + & & \\
\hline
\end{tabular}


Table 2 (Contd.)

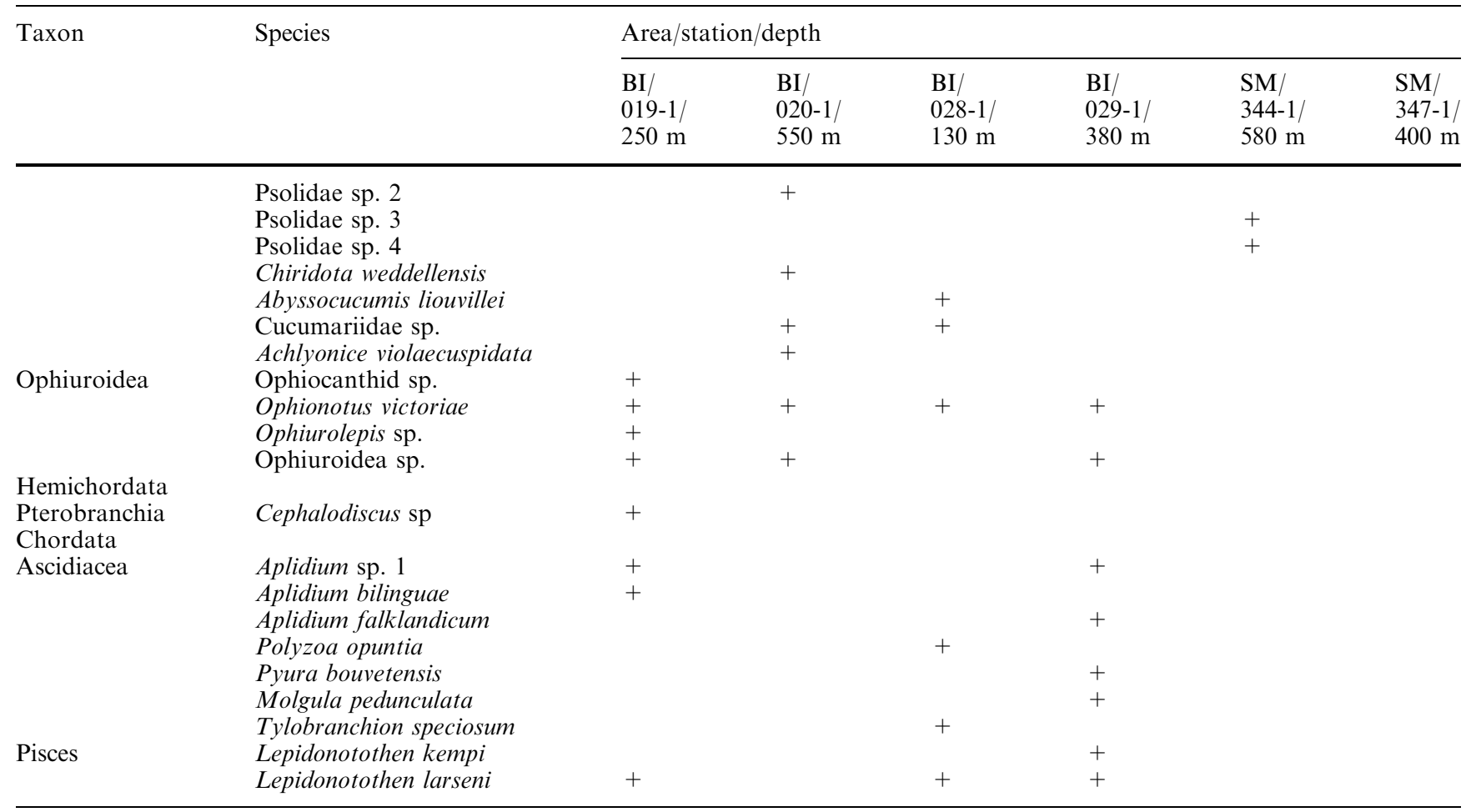

Only one undetermined Harmothoe polychaete was found in the Spiess Seamount samples (Table 2).

Mollusca Molluscs belong to the more species-rich group, with 25 shelled and 7 non-shelled species of gastropods, 12 species of bivalves, 3 species of polyplacophorans, and 1 species of solenogastre (Aplacophora). The species composition of the shelled malacofauna is described in detail by Linse (2005). Nudibranchia, not reported before, include the circum-Antarctic species Austrodoris kerguelensis, Notaeolidia depressa, Tritonia challengeriana, and $T$. dantari sp. n. (for description, see Ballesteros and Avila 2005) as well as undescribed species of Doto sp. and Cuthona sp..

Chelicerata-Pycnogonida Four species of sea spiders were found at Bouvet Island, of which Nymphon australe with five male and four female collected specimens was most abundant (as occurs in high Antarctic areas). Both sexes of Colossendeis robusta were also collected while only one male of Pallenopsis hodgsoni and two juveniles of Ammothea carolinensis were found. C. robusta and P. hodgsoni are reported here for the first time for Bouvet Island.

Crustacea-Malacostraca Amphipoda. One baited trap deployment and four trawling and dredging operations around Bouvet Island allowed the collection of about 1,200 specimens of amphipod crustaceans, attributed to more than 60 amphipod species (Table 2). Previously, only five benthic amphipod species were known from the area. Among these 60 species, 1 genus and 14 species belonging to the families or superfamilies Eusiroidea (1 sp.), Iphimediidae (2 spp.), Liljeborgiidae (2 spp.), Lysianassoidea (1 genus, 3 spp.), and Stenothoidae (6 spp.) appear to be new to science (Rauschert 2005). The species of Lysianassoidea, Abyssorchomene plebs and Abyssorchomene sp., and Orchomenopsis sp. were found in the baited traps only. The biogeographical affinities of the Bouvet amphipod fauna, as presently known (on the basis of 52 fully identified species), appear to be strongest with the West Antarctic fauna (Antarctic Peninsula and Scotia Arc, including South Georgia): $36 \%$ of Bouvet species also occur in the West Antarctic and 14\% are present in South Georgia. The species share with the East Antarctic fauna amounts to $29 \%$ and with the Weddell Sea alone to $18 \%$.

Isopoda. The 13 isopod species/morphotypes distinguished so far mainly represent the Weddell Sea fauna and thus support a strong biogeographic relationship with the high Antarctic (Table 2).

Decapoda. Decapod crustaceans were rare around Bouvet, with only three hippolytid shrimp species found in single specimens (Table 2). Whereas two species so far appear to be unknown to science and require further taxonomic analyses, the single record of Nauticaris marionis, commonly known from waters around Marion Island, extends the distribution of this species south and across the Polar Front (see Gorny 1999). Nematocarcinus lanceopes is a typical deep-water to deep-sea shrimp 
Fig. 5 Pie charts of macrozoobenthic composition for 51 AGT subsamples
PS 65/028-1, 130m

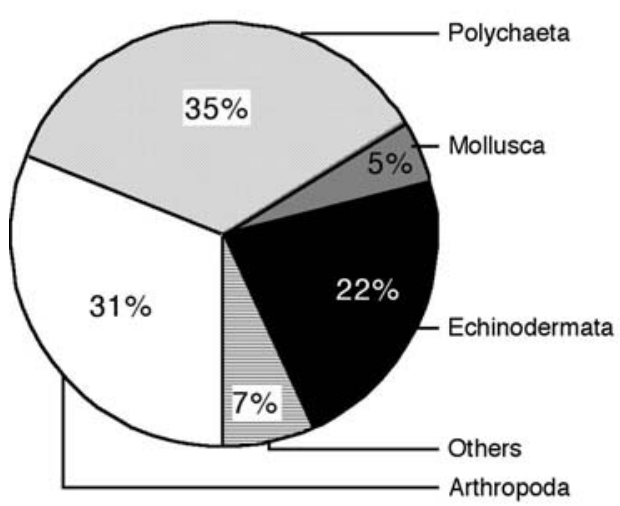

PS 65/029-1, 380m

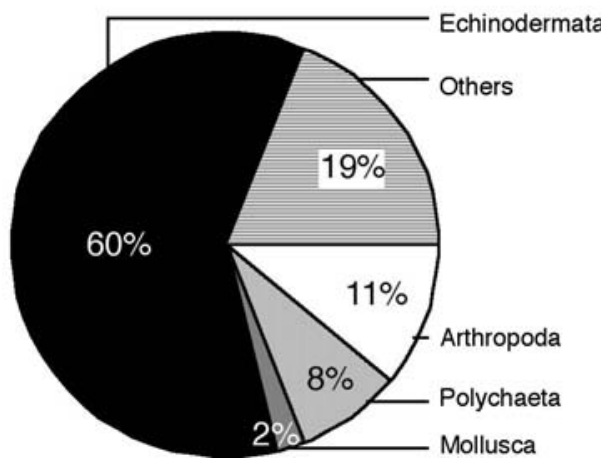

PS 65/019-1, 250m

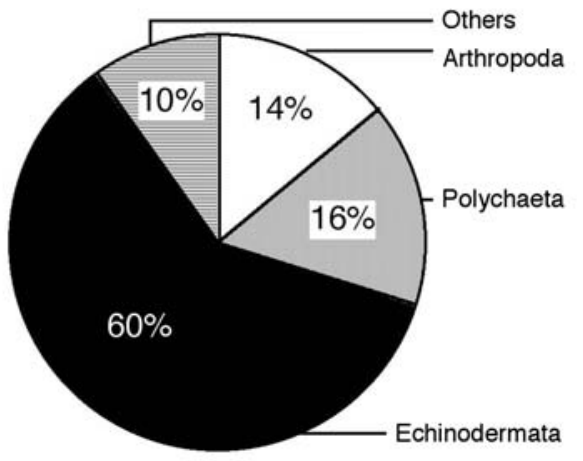

PS $65 / 020-1,550 m$

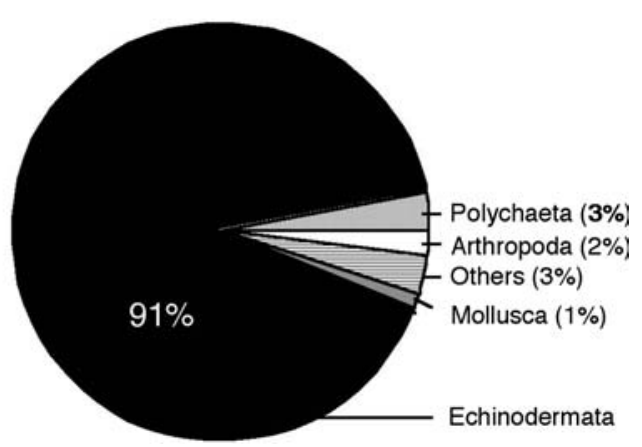

of the Southern Ocean. Its presence at the Spiess Seamount clearly extends its distribution northwards (Table 2). Paralomis elongata sp. n. (Spiridonov et al. 2005 ) is the only anomuran crab found at the Spiess Seamount. Its presence not only in a few trawls, but also on the underwater images, indicates this stone crab to be an important faunistic element of the seamount benthos (Fig. 3, Table 2).

Bryozoa A total of 18 species of cheilostomes were collected at the four sites of which only four had been reported before. Next to the 14 new species records, 8 genera were reported for Bouvet Island for the first time (Barnes 2005).

Echinodermata Most echinoderm species found remain for further determination to species level. Crinoids and echinoids have been very rare in our samples, especially echinoids were represented by single specimens only; holothurians occurred in dense patches at station PS65/ 024-1, at about $250 \mathrm{~m}$ water depth (Fig. 3). Asteroidea were found at all stations (at Spiess Seamount from underwater images mainly; Figs. 3, 4). Ophiuroids by far outnumbered any other taxon found in our samples, including at Spiess Seamount (Figs. 3, 4).

Ascidiacea Ascidians were poorly represented in the samples with 17 specimens (solitary and colonial organisms) comprising seven species (Table 2). Previously, only four species were known from Bouvet Island (Michaelsen 1904; Millar 1960, 1968; Kott 1969; Monniot and Monniot 1983): Polyzoa opuntia, Cnemidocarpa verrucosa, Pyura bouvetensis, and Molgula pedunculata. Three new records have contributed to enlarge the Bouvet's fauna: Aplidium bilinguae, A. falklandicum, and Tylobranchion speciosum. Nevertheless, the ascidian fauna at Bouvet remains poor with only 7 species known so far, in comparison to $>50 \mathrm{spp}$. from other Subantarctic islands (such as South Georgia and Kerguelen Islands). From a biogeographical point of view, Bouvet's ascidiofauna participates from species with a wide Antarctic-Subantarctic eurybathic distribution ( $A$. falklandicum, $T$. speciosum, $C$. verrucosa, $P$. bouvetensis, and $M$. pedunculata) and a littoral Subantarctic component (P. opuntia). A. bilinguae has been previously recorded from the Antarctic continent (Wilkes' Land) and the island of South Georgia (Monniot and Monniot 1983).

Demersal fish At Bouvet Island the nototheniid L. larseni was highly abundant and the only species found between 130 and $250 \mathrm{~m}$ water depth. In one haul at $370 \mathrm{~m}$ a single individual of Lepidonotothen kempi was caught besides L. larseni. Since both species are common constituents of the southern Scotia Arc fauna, dispersion via larval drift within the Circumpolar Current is very likely. 
The data collected by AGT and RD at the same stations (Table 2) were pooled for the diversity and multidimensional analysis. The combined taxon presence data show that station PS65/028-1 was the most taxon rich (98 spp.), followed by PS65/029-1 (90 spp.) and PS65/019-1 (76 spp.) (Table 2). The multivariate analysis of the Bray-Curtis similarity (Bray and Curtis 1957), based on the presence/absence, showed clear patterns of benthic communities (Fig. 6). The two samples from the Spiess Seamount group together and far apart from the Bouvet samples. Of the four stations collected on the Bouvet shelf, PS65/019-1 and PS65/0291 group closely together, whereas PS65/020-1 and PS65/ 028-1 plot apart from any station.

\section{Discussion}

In some taxa the number of species known for Bouvet Island increased substantially. Notable examples were the benthic amphipods (from 5 to $62 \mathrm{spp}$., of which at least 7 were new to science; see also Rauschert 2005a, b), the molluscs (from 16 to 45 spp.; see Linse 2005), and the bryozoans (from 20 to 34 spp.; see Barnes 2005). If these findings are representative of other taxa, such steep increases suggest that a major reason for the impoverished fauna lists for Bouvet's surrounding waters is undersampling rather than isolation or geological youth. Only when species accumulation curves start approaching asymptote, can strong conclusions about the biodiversity or biogeography of the area begin to be drawn. Gauging the richness and particular taxa presence at isolated, young rises such as Bouvet and Spiess is very useful as some (such as peracarid crustaceans, most bryozoans, and molluses) do not disperse easily, having effectively benthic or brooded larvae. By comparing the number of colonist taxa with their larval strategy types and allowing for the nearest known localities, we can get a much better picture of how colonisation of the many islands surrounding Antarctica has occurred. Existing data, prior to species asymptotes, enable some useful insight, however, into the relative rate of finding species new to Bouvet versus those new to science. Bouvet Island is very young to have many endemics but conversely is very isolated, which promotes endemism.

In contrast to Bouvet, Agassiz trawls at the Spiess Seamount $(570 \mathrm{~m})$ yielded very little material. Samples did, however, include three specimens of a lithodid decapod, one of which was an egg-carrying female with prezoeae close to hatching (Paralomis elongata $\mathrm{n}$. sp.; Spiridonov et al. 2005). Furthermore, five deep-sea carideans ( $\mathrm{Ne}$ matocarcinus lanceopes) were collected, at their most shallow depths known to date (Gorny 1999).

The biogeographic relations of the deeper shelf fauna around Bouvet were compared with those of high Antarctic, Antarctic Peninsula, and Magellanic samples taken during previous Polarstern cruises. We did not consider nearshore shallow water and deep sea due to lack of sampling from these areas. The general composition of the Bouvet fauna known to date is more similar to Magellanic South America rather than the high Antarctic region. Affinities of the motile peracarids and the molluscs (except for nudibranchs) had most linkages with the faunas of the Antarctic Peninsula and the Scotia Arc. Such a distribution could well be explained by the transport of adults, larvae, and drift stages via the West Wind Drift/Circumpolar Current (see also Gutt et al. 2005).

Some taxa may show considerable eurybathy; we found the gastropod Calliotropis (Solaricida) antarctica at $270 \mathrm{~m}$, but it had previously been found at $775 \mathrm{~m}$ on an ANDEEP cruise and at 2,700 $\mathrm{m}$ at the Antarctic Peninsula (Linse et al. 2003). Those taxa more closely related to the high Antarctic fauna, such as the nudibranchs, hydrozoans, gorgonians, and ascidians, might have dispersed to Bouvet Island via the Weddell Gyre. The decapods of the Bouvet/Spiess region reveal both Scotia Arc relations (lithodids, hippolytids) and Weddell Sea affinities ( $N$. lanceopes). We suggest that, except for the hippolytid decapods, colonisation may have
Fig. 6 Bouvet Island and Spiess Seamount macrofauna. MDS ordination of the six sites based on the presence/absence data and Bray-Curtis similarities. Stations 344-1 and 347-1 represent samples taken on the Spiess Seamount

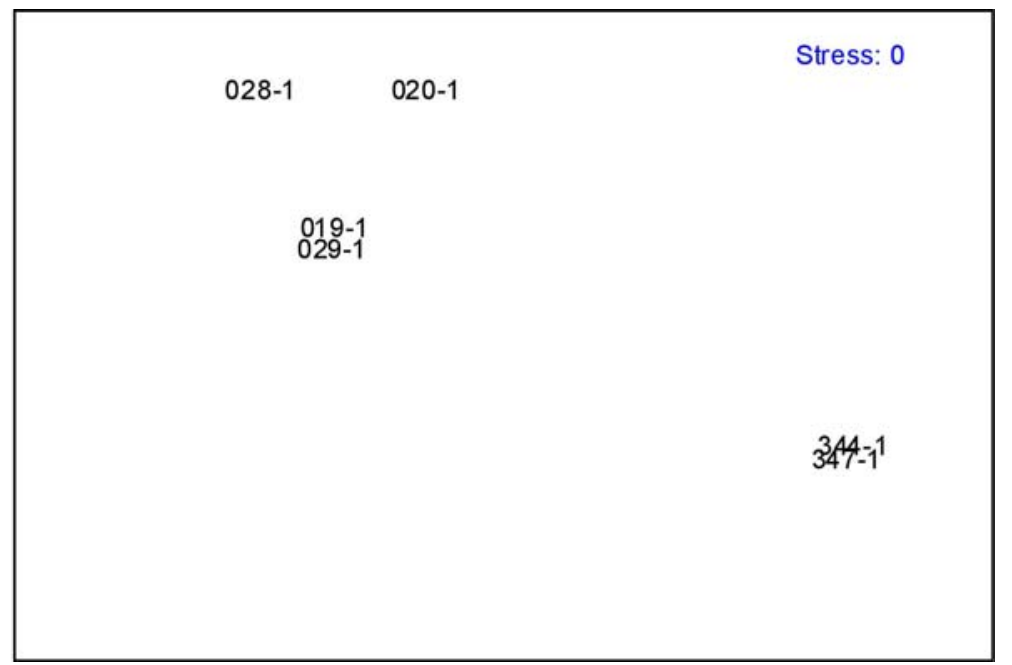


occurred via the deep sea (Thatje et al. 2005a). Of the fish, the two Lepidonotothen species are characteristic species of the Scotia Arc (e.g. Kock and Stransky 2000), but juveniles of $L$. kempi were also found in the $\mathrm{E}$ Weddell Sea. It is unknown whether the different populations have maintained active exchange. Material of other taxa is currently under analysis by specialists, who may be able to suggest most likely respective affiliations. Some of these biogeographic (and possibly, phylogenetic) relationships will be further elucidated after the analysis of the large material to be subjected to molecular genetic techniques at the home laboratories. It should also be mentioned that both waters off Bouvet Island and Spiess Seamount have been fished in the past and therefore the benthos may have been impacted. Thus, the effects of altered benthic communities on this biogeographic analysis remain uncertain.

Acknowledgements The participants of RV "Polarstern" cruise ANT XXI/2 would like to express their sincere gratitude to the Norwegian Government for granting permission to study the fauna in the Bouvet region. Our thanks go to the Master of RV "Polarstern", Udo Domke, and his crew for competent and friendly assistance on board. The valuable help of Harald Bohlmann with the CTD management is gratefully acknowledged. We would like to thank Pilar Ríos (Univ. of Santiago de Compostela, Spain) for helping in the Porifera identification. The map was provided by $\mathrm{H}$. Griffiths (BAS). The useful comments by three anonymous reviewers are greatly acknowledged.

\section{References}

Arntz WE, Brey T (2001) The Expedition ANTARKTIS XVII/3 (EASIZ III) of RV "Polarstern" (Eds). Ber Polar Meeresforsch (Rep Polar Mar Res) 402:1-181

Arntz WE, Brey T (2003) The Expedition ANTARKTIS XIX/5 (LAMPOS) of RV "Polarstern" in 2002 (Eds). Ber Polar Meeresforsch (Rep Polar Mar Res) 462:1-120

Arntz WE, Brey T (2005) The Expedition ANTARKTIS XXI/2 (BENDEX) on RV "Polarstern" in 2003/2004 (Eds). Ber Polar Meeresforsch (Rep Polar Mar Res) 503:1-149

Arntz WE, Gorny M (1996) Cruise report of the Joint ChileanGerman-Italian Magellan "Victor Hensen" Campaign in 1994 (Eds). Ber Polarforsch (Rep Polar Res) 190:1-113

Arntz WE, Gutt J (1997) The expedition ANT XIII/3 (EASIZ I) of the "Polarstern" to the eastern Weddell Sea in 1996 (Eds). Ber Polarforsch (Rep Polar Res) 249:1-148

Arntz WE, Gutt J (1999) The Expedition ANTARKTIS XV/3 (EASIZ II) of RV "Polarstern" in 1998. Ber Polarforsch (Rep Polar Res) 301:1-229

Arntz WE, Ríos C (1999) Magellan-Antarctic: ecosystems that drifted apart (Eds). Sci Mar 63 (Suppl 1):1-518

Arntz WE, Lovrich GA, Thatje S (2005) The Magellan-Antarctic connection: links and frontiers at high latitudes (Eds). Sci Mar (Suppl) (in press)

Ballesteros M, Avila C (2005) A new tritoniid species (Mollusca: Opisthobranchia) from Bouvet Island. Polar Biol (this volume)

Barber HN, Dadswell HE, Ingle HD (1959) Transport of driftwood from South America to Tasmania and Macquarie Island. Nature 184:203-204

Barnes D (2002) Invasions by marine life on plastic debris. Nature 416:808-809

Barnes DKA (2005) A most isolated benthos: coastal bryozoans of Bouvet Island. Polar Biol (this volume)

Barnes DKA, Warren N, Webb K, Phalan B, Reid K (2004) Polar pedunculate barnacles piggy-back on pycnogona, penguins, pinniped seals and plastics. Mar Ecol Prog Ser 284:305-310
Bergenhayn JRM (1937) Antarktische und subantarktische Polyplacophoren. Sci Res Norw Antarctic Expedit 1927-1928 17:112

Beu AG, Griffin M, Maxwell PA (1997) Opening of Drake Passage gateway and late Miocene to Pliocene cooling reflected in Southern Ocean molluscan dispersal: evidence from New Zealand and Argentina. Tectonophysics 282:83-97

Bray JR, Curtis JT (1957) An ordination of the upland forest of Southern Wisconsin. Ecol Monogr 27:225-349

Brey T, Dahm C, Gorny M, Klages M, Stiller M, Arntz WE (1996) Do Antarctic benthic invertebrates show an extended level of eurybathy? Antarctic Sci 8:3-6

Clarke A, Johnston NM (2003) Antarctic marine benthic diversity. Oceangr Mar Biol Annu Rev 41:47-114

Clarke KR, Warwick RM (2001) Change in marine communities: an approach to statistical analysis and interpretation. PRIMER-E Ltd, Plymouth

Coombs DS, Landis CA (1966) Pumice from the South Sandwich eruption of March 1962 reaches New Zealand. Nature 209:289290

Dell RK (1964) Antarctic and sub-Antarctic Mollusca: Amphineura, Scaphopoda and Bivalvia. Discov Rep 23:93-250

Desqueyroux R, Moyano H (1987) Zoogeografía de demospongias chilenas. Bol Soc Biol Concepción 58:39-66

Engelskjön T (1981) Terrestrial vegetation of Bouvetøya. A preliminary account. Norsk Polarinst Skr 175:17-28

Fevolden SE (1980) Krill off Bouvetøya and in the southern Weddell Sea with a description of larval stages of Euphausia crystallorophias. Sarsia 65:149-162

Furnes H, Løvlie R (1978) An eruptional model for the recent lava flow on Bouvetøya, South Atlantic Ocean. Norsk Polarinst Skr 169:103-108

Gayoso AM, Podestá GP (1996) Surface hydrography and phytoplankton of the Brazil-Malvinas currents confluence. J Plankton Res 18:941-951

Glorioso PD, Piola AR, Leben RR (2005) Mesoscale eddies in the Subantarctic Front-Southwest Atlantic. Sci Mar (Suppl) (in press)

Gorny M (1999) On the biogeography and ecology of the Southern Ocean decapod fauna. Sci Mar 63 (Suppl 1):367-382

Gutt J, Sirenko BI, Arntz WE, Smirnov IS, De Broyer C (2000) Biodiversity of the Weddell Sea: macrozoobenthic species (demersal fish included) sampled during the expedition ANT XIII/3 (EASIZ I) with RV 'Polarstern'. Ber Polarforsch (Rep Polar Res) 372:1-103

Gutt J, Fricke A, Teixidó N, Potthoff M, Arntz WE (2005) Megaepibenthos at Bouvet Island: a spatially isolated biodiversity hot spot on a tiny geological hot spot. Polar Biol (this volume)

Haftorn S, Somme L, Gray JS (1981) A census of penguins and seals on Bouvetøya. Skr Norsk Polarinst 175:29-35

Helmuth B, Veit RR, Holberton R (1994) Long-distance dispersal of a subantarctic brooding bivalve (Gaimardia trapesina) by kelp rafting. Mar Biol 120:421-426

Holdgate MW, Tilbrook PJ, Vaughan RW (1968) The biology of Bouvetøya. Br Antarctic Surv Bull 15:1-7

Kock K, Stransky C (2000) The composition of the coastal fish fauna around Elephant Island (South Shetland Islands, Antarctica). Polar Biol 23:825-832

Kott P (1969) Antarctic Ascidiacea II. Antarctic Res Ser 17:11-82

Linse K (2005) New records of marine molluscs at Bouvet Island and their biogeographic affinities. Polar Biol (this volume)

Linse K, Schrödl M, McClain CR, Allcock L (2003) Mollusca in the Antarctic deep sea-preliminary notes on their taxonomy, biogeography and diversity. In: Fütterer DK, Brandt A, Poore GCB (eds) The Expeditions ANTARKTIS-XIX/3-4 of the Research Vessel POLARSTERN in 2002. Ber Polar Meeresforsch (Rep Polar Mar Res) 470:95-101

Michaelsen W (1904) Die stolidobranchiaten Ascidien der deutschen Tiefsee-Expedition. Wiss Ergeb Dtsch Tiefsee-Expedit $7(2): 181-260$

Millar RH (1960) Ascidiacea. Discov Rep 30:1-160 
Millar RH (1968) Ascidians collected during 1928-1930 by the Norwegian Antarctic expeditions. Det Norsk Videnskaps-Akad Oslo I: Mat-Naturv Klasse 10:3-25

Monniot C, Monniot F (1983) Ascidies antarctiques et subantarctiques: morphologie et biogéographie. Mem Mus Natl Hist Nat Paris 215(Sér A): $1-135$

O Foighil D, Marshall BA, Hilbish TJ, Pino MA (1999) TransPacific range extension by rafting is inferred for the flat oyster Ostrea chilensis. Biol Bull 196:122-126

Perissinotto R, Laubschner RK, McQuaid CD (1992) Marine productivity enhancement around Bouvet and the South Sandwich Islands (Southern Ocean). Mar Ecol Prog Ser 88:4153

Powell AWP (1951) Antarctic and sub-Antarctic Mollusca: Pelecypoda and Gastropoda. Discov Rep 26:49-196

Prestvik T, Winsnes TS (1981) Geology of Bouvetøya, South Atlantic. Skr Norsk Polarinst 175:41-68

Rauschert M (2005) Atyloella tribinicuspidata sp. n. (Crustacea, Amphipoda, Gammaridea, Eusiridae), a common amphipod in benthic communities off Bouvet Island (Southern Ocean). Polar Biol (this volume)

Somme L (1986) Terrestrial arthropods of Bouvetøya. Polar Res 4:231-233

Soot-Ryen T (1951) Antarctic Pelecypoda. Det Norsk VidenskapsAkad Oslo Sci Res Norw Antarctic Expedit (1927-1929) 32:144
Spiridonov V, Türkay M, Arntz WE, Thatje S (2005) A new species of the genus Paralomis (Crustacea: Decapoda: Lithodidae) from the Spiess Seamount near Bouvet Island (Southern Ocean), with notes on habitat and ecology. Polar Biol (this volume)

Thatje S, Fuentes V (2003) First record of anomuran and brachyuran larvae (Crustacea: Decapoda) from Antarctic waters. Polar Biol 26:279-282

Thatje S, Anger K, Calcagno JA, Lovrich GA, Pörtner HO, Arntz WE (2005a) Challenging the cold: crabs reconquer the Antarctic. Ecology 86:619-625

Thatje S, Linse K, Arntz WE (2005b) Bouvet Island-a stepping stone in the Southern Ocean? Polar Biol (this volume)

Thiele J (1904) Die beschalten Gastropoden der Deutschen TiefseeExpedition 1898-1899. B. Anatomisch-systematische Untersuchungen einiger Gastropoden. In: Chun C (ed) Wiss Ergeb Dtsch Tiefsee-Expedit Dampfer "Valdivia“ (1898-1899):149179

Thiele J (1925) Gastropoda der Deutschen Tiefsee-Expedition 1898-1899. In: Chun C (Ed) Wiss Ergeb Dtsch Tiefsee-Expedit Dampfer "Valdivia" (1898-1899):338-348

Thiele J, Jaeckel S (1931) Muscheln der deutschen Tiefsee-Expedition. Dtsch Tiefsee-Expedit 1898-1899 XXI:160-268 